\title{
Comparative Effectiveness of Mild or Conventional GnRH-Antagonist Protocols for Ovarian Stimulation in Poor Responders (Poseidon Group 4)
}

\author{
Hoang Le ${ }^{1 *}$, Dong D. Nguyen ${ }^{1}$, Anh T. Cao ${ }^{1}$, Huong T. L. Nguyen ${ }^{1}$, Dung C. Tham ${ }^{1}$, \\ Thang D. Le ${ }^{1}$ and Jean-Noël Hugues ${ }^{2,3 *}$ \\ ${ }^{1}$ IVFTA, Tam Anh General Hospital, Hanoi, Vietnam, ${ }^{2}$ Department of Obstetrics, Gynecology and Reproductive Medecine, \\ Hôpitaux Universitaires Paris Seine Saint-Denis, Assistance Publique-Hôpitaux de Paris, Bondy, France, ${ }^{3}$ Université Paris 13, \\ Sorbonne Paris Cité, UFR SMBH, Bobigny, France
}

OPEN ACCESS

Edited by:

Johnny S. Younis,

The Baruch Padeh Medical

Center, Israel

Reviewed by:

Nikolaos P. Polyzos,

Dexeus University Hospital, Spain

Janice Vilela,

Catholic University of

Louvain, Belgium

*Correspondence: Jean-Noël Hugues

jnhugues@yahoo.fr

Hoang Le

lehoang@tamanhhospital.vn

Specialty section:

This article was submitted to

Assisted Reproduction,

a section of the journal

Frontiers in Reproductive Health

Received: 14 September 2020 Accepted: 10 November 2020 Published: 04 December 2020

Citation:

Le H, Nguyen DD, Cao AT, Nguyen HTL, Tham DC, Le TD and Hugues J-N (2020) Comparative Effectiveness of Mild or Conventional GnRH-Antagonist Protocols for

Ovarian Stimulation in Poor Responders (Poseidon Group 4). Front. Reprod. Health 2:606036. doi: 10.3389/frph.2020.606036
Background and Aims: A panel of experts (the Poseidon Group) introduced a new and more detailed stratification for poor ovarian responders in order to predict the prognosis of IVF outcome according to the sensitivity to FSH. However, various arguments about the management strategy of these patients still remain, including the convenience and the cost. Therefore, this study was conducted to compare the efficacy of mild and conventional $\mathrm{GnRH}$ antagonist ovarian stimulation prescribed in patients classified in Poseidon Group 4.

Methods: This retrospective cohort study included 359 poor responder patients (Poseidon Group 4) treated with mild or conventional $\mathrm{GnRH}$ antagonist stimulation regimens from 8/2017 to 7/2019 at Tam Anh Hospital ART Center. The main outcomes were the index of Follicular Output Rate (FORT) or Follicle to Oocyte Index (FOI), the number of day-2 embryos and top-quality embryos obtained. The $t$-test and Mann-Whitney $U$ test in SPSS v25.0 was used to analyze the continuous data and Chi-squared/Exact test was used for binary variables. Multiple linear regression analysis was done by using Stata versions 15.0 to measure association between primary endpoints with stimulation regimen controlled for covariates and possible confounding factors.

Results: In the overall group of poor responders, the conventional $\mathrm{GnRH}$ antagonist protocol performed better than the mild protocol. Subsequently, data were analyzed according to the AFC. In women with AFC $<3$, no significant differences were observed between the 2 regimens regarding FORT $(p=0.71)$, FOI $(p=0.12)$, the number of day-2-embryos $(p=0.052)$ and the number of top-quality embryos $(p=0.26)$. In contrast, in women with AFC $\geq 3$, mild stimulation regimen resulted in significantly poorer outcome compared to the conventional $\mathrm{GnRH}$ antagonist regimen, regarding FORT $(p<0.01)$, FOI $(p<0.01)$, the number of day-2-embryos $(p<0.01)$ and top-quality embryos $(p=0.01)$.

Conclusions: Considering poor responders classified in Poseidon Group 4, both ovarian stimulation regimens resulted in similar outcome for patients with a very low ovarian reserve (AFC < 3). In contrast, the $\mathrm{GnRH}$ conventional antagonist protocol with maximum initial FSH dose (300-375 IU/day) and supplementary LH (75-150 IU/day) was 
more effective than the mild one for patients whose ovarian reserve was less reduced. The Clinical Trial was approved by the Ethnical Biomedical Research Committee Tam Anh General Hospital.

Keywords: Poseidon, mild stimulation, poor responders, GnRH antagonist, IVF

\section{BACKGROUND}

In contemporary times, as women tend for bearing children at older age, the average age of first-time mothers is increasing in many countries (1). Thus, an increasing number of women facing a decreased ovarian reserve are asking for assisted reproductive technology to get a child (2). This subgroup represents about $37 \%$ of the overall IVF population (3), even if prognosis is very poor with a live birth rate between 6.7 and $11.4 \%$ (48).

Management and treatment strategies for patients displaying a poor response (POR) to ovarian stimulation are still in debate. One of the reasons is related to the heterogeneity of this subgroup of patients. Due to the lack of consensus regarding definition of POR [41 different definitions in 47 RCT (9)], some experts suggested at a Bologna meeting in 2011 a new definition based on age, ovarian reserve and previous response to stimulation (10). However, as 2 out of 3 criteria could be used to define a POR, several types of patients with different prognosis factors could be integrated within this category. To overcome those limitations of Bologna criteria, a group of scientists/clinicians published in 2016a new classification called Poseidon (Patient-Oriented Strategies Encompassing IndividualizeD Oocyte Number) aiming at defining some subgroups with different prognosis according to their ability to get at least one euploid blastocyst for transfer (11). Besides the issue of definition, no consensual therapeutic strategy could emerge until now $(12,13)$. Increasing gonadotropin dose over a certain threshold is actually ineffective (14-17). A survey involving 196 ART centers in 45 nations showed that the most common protocol included $\mathrm{GnRH}$ antagonist regimen (53\%), rFSH and hMG (43\%) at a dose of 300-375 IU per day (36.7\%), while $37 \%$ of centers applied mild ovarian stimulation protocol which combines Clomiphene citrate and FSH (13). AFC and $\mathrm{AMH}$ are efficient markers to predict both poor and hyper ovarian response but they are just "still images" and cannot reflect the dynamic of follicular growth induced by FSH. Consequently, it has been suggested to assess the actual response to FSH by measuring Follicular Output Rate (FORT) and Follicle to Oocyte Index (FOI) which both reflect the follicular sensitivity to FSH $(18,19)$. Improving the ovarian response to FSH is actually a key issue to enhance the reproductive prognosis of Poseidon groups 3 and 4 patients (18). Indeed, FOI improvement should increase the chance of having euploid embryos and the success rate (19).

Regarding patients classified in Poseidon group 4, an individualized treatment was recommended using $\mathrm{GnRH}$ antagonist protocol with the maximum starting dose of $\mathrm{rFSH}$ is 300 IU per day (11) alone or in association with rLH (75-150 IU per day) (20, 21). Another more cost-effective option could be the prescription of a mild stimulation. While no significant difference between mild stimulation and conventional GnRH antagonist regimen could be demonstrated regarding oocyte and embryo numbers and the clinical pregnancy rate, it might be explained by the differences in POR definition and patient selection criteria (22-24). Therefore, the objective of our study was to compare the efficacy of mild and conventional GnRH antagonist protocols in patients classified in Poseidon group 4. For that purpose, both FORT and FOI index were used to assess the ovarian response to gonadotropins.

\section{MATERIALS AND METHODS}

\section{Patients}

This retrospective cohort study was conducted in IVFTA, Tam Anh General Hospital, from August 2017 to July 2019. All women classified as POR [group 4 of Poseidon classification: age $\geq 35$ yrs, poor ovarian reserve $(\mathrm{AFC}<5, \mathrm{AMH}<1.2 \mathrm{ng} / \mathrm{ml})$ ] were eligible. All patients who had alternative illness which could affect oocyte stimulation cycle, congenital uterine abnormalities, ovulatory disorders, abnormal sperm were excluded from the analysis.

\section{Ovarian Stimulation, Oocyte Retrieval, Zygote and Embryo Assessment, Luteal Support}

All patients were prescribed one of two follicular stimulation protocols: Mild stimulation (100 mg Clomiphene Citrate from day 2 to day 7, hMG 75 IU per day from day 7) or conventional stimulation (FSH 300-375 IU per day + r.LH 75-150 IU per day from day 2).

In both protocols, GnRH antagonist was added from day 6 of ovarian stimulation. Ovulation was triggered using 10,000 IU of hCG (Pregnyl, Schering - Plough Organon, Oss, the Netherlands) when the leading follicle diameter reached $18 \mathrm{~mm}$. Oocyte retrieval was performed $34-36 \mathrm{~h}$ after triggering ovulation. The cycle had to be canceled if there was no developing follicle or if follicular size was $<15 \mathrm{~mm}$ after 7 days of stimulation. Embryo transfer was performed with 1 or 2 good quality cleave-stage (day 3) embryos. Transfer of more than two embryos was allowed if the woman was over 40 or if the embryo quality was not good. Supernumerary embryos were frozen and transferred in subsequent cycle.

The morphological score, the cell number, degree of fragmentation of the embryo and the uniformity of the blastomeres were assessed daily. Embryo quality were classified 
into grade I (no fragmentation), grade II ( $<20 \%$ fragmentation), grade III (20-50\% fragmentation) and grade IV ( $>50 \%$ fragmentation). Good quality embryos included grade I and grade II embryos; Poor quality embryos included grade III and grade IV embryos. After embryo transfer, luteal support treatment was prescribed immediately until at least 12 -week of pregnancy; all patients received Dydrogesterone $30 \mathrm{mg} /$ day (Dusphaton ${ }^{\circledR} 10 \mathrm{mg}$, Abott, t.i.d. orally) and vaginal or anal suppository micro-Progesterone $600 \mathrm{mg} /$ day (age $<38 \mathrm{yrs}$ ) or $800 \mathrm{mg}$ /day (age $\geq 38 \mathrm{yrs}$ ) (Utrogestan ${ }^{\circledR} 200 \mathrm{mg}$, t.i.d. vaginal suppository or Cyclogest ${ }^{\circledR} 200 \mathrm{mg}$ vaginal/anal suppository t.i.d. or Cyclogest ${ }^{\circledR} 400 \mathrm{mg}$ vaginal/anal suppository b.i.d.) Measurement of serum beta hCG concentration was performed on day 12-14 post transfer and ultrasound examination to detect a gestational sac on day 21. Biochemical pregnancy (positive beta hCG in blood or urine without gestational sac at ultrasound), clinical pregnancy (presence of gestational sac including ectopic pregnancy), early pregnancy loss (miscarriage or stopped developing fetus before 12 weeks) and ongoing pregnancy (pregnancy with developed fetal heart activity till minimum 12 weeks of gestation) were all recorded.

\section{End Points}

The primary end points of our study were the following: FORT (Follicular Output Rate: calculated as the ratio of pre-ovulatory follicle count (from 16 to $22 \mathrm{~mm}$ in diameter) on day of triggering/Antral follicle count under transvaginal ultrasound scans), FOI (Follicle to oocyte index: the ratio of the number of retrieved oocyte/antral follicle count), and the number of day-2 embryos, good quality embryos.

The secondary end points were the ongoing pregnancy, clinical pregnancy, early pregnancy loss, biochemical pregnancy, ovarian stimulation duration, total gonadotropin dose, ratio of the canceled cycles, ratio of fertilization.

\section{Statistical Analysis}

SPSS (version 25.0, http://ibm-spss-statistics.com) and Stata, version 15.0 (Stata Corp, 2017, TX) for statistical analysis were used. The difference of binary outcomes was measured by using relative risk (RR) with $95 \%$ confidence interval and testing the differences was evaluated according to Fisher Exact test or Chi-square test as appropriate. For the remaining variables, the mean and standard deviation were also estimated; and compared their differences by using two-tailed $t$ test or Mann - Whitney U test methods. Multiple linear regression analysis method was applied to evaluate the association between the performance of FOI, FORT, Day 2 embryos and Topquality embryos as continuous outcomes and Mild/Conventional protocol in each group of AFC ( $<3$ vs. $\geq 3$ ) as conditional. The linear regression models were adjusted for possible covariates or confounding factors including age in year, BMI, AMH, duration of infertility in year, primary infertility. The stepwise regression of multiple variables was applied aiming at simultaneously remove the weakest correlated variables which did not significantly influence the adjusted $\mathrm{R}^{2}$ in the model. Level of confident was at $5 \%$.

\section{RESULTS}

From August 2017 to July 2019, 359 patients were eligible, 186 patients receiving the mild stimulation protocol and 173 patients receiving the conventional GnRH antagonist protocol (Figure 1). Twenty-five patients had cycles canceled or stopped at will. The remaining 334 patients were included in the analysis, consisting

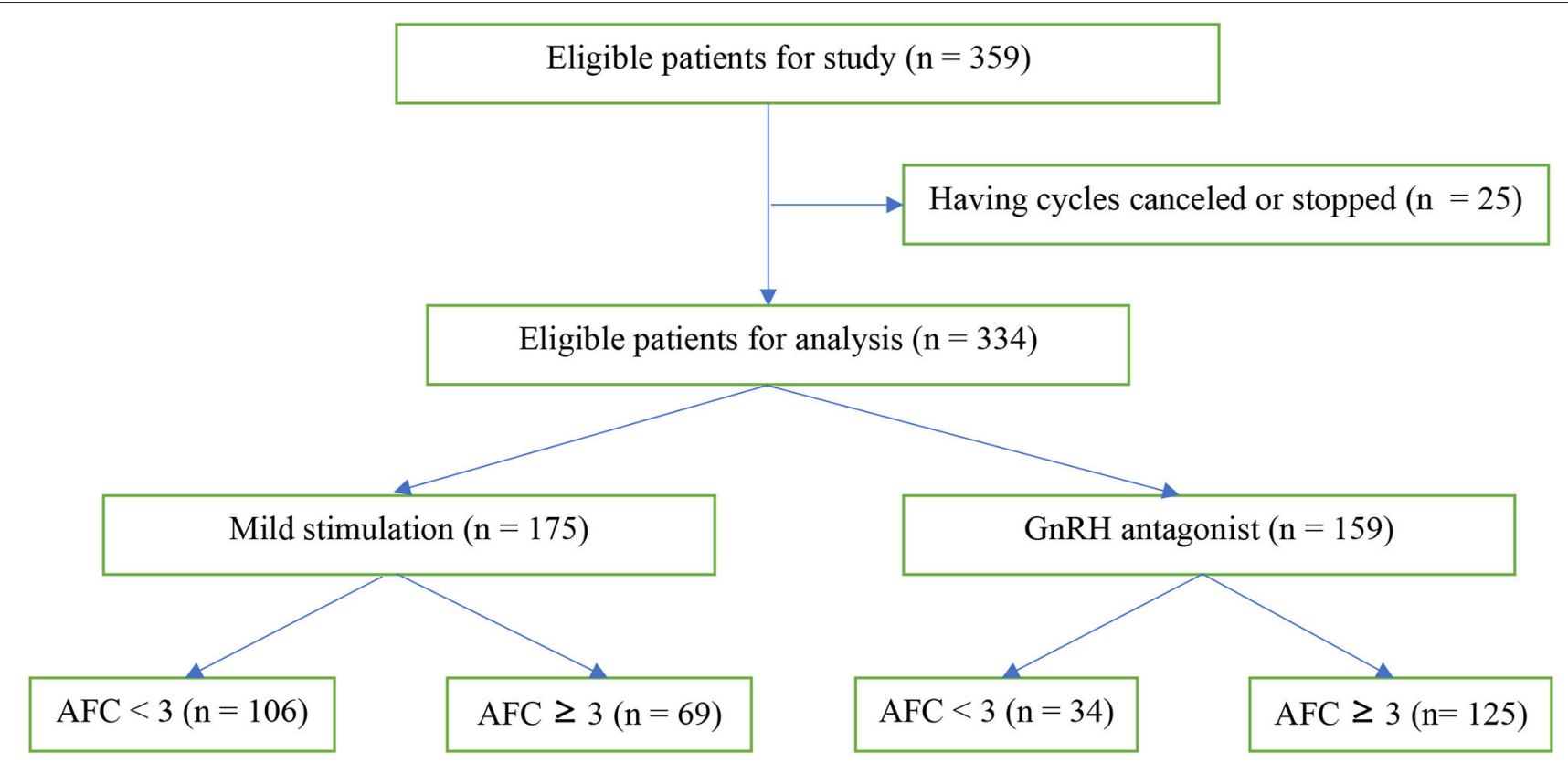

FIGURE 1 | Study flow diagram. 
TABLE 1 | Baseline characteristics and outcome of patients treated with Mild $(n=175)$ and Conventional protocols $(n=159)$.

\begin{tabular}{lccc}
\hline & $\begin{array}{c}\text { Mild } \\
(\boldsymbol{n}=\mathbf{1 7 5})\end{array}$ & $\begin{array}{c}\text { Conventional } \\
(\boldsymbol{n}=\mathbf{1 5 9})\end{array}$ & $\boldsymbol{p}$-value \\
\hline Age (yrs) & $42.1 \pm 3.6$ & $41.0 \pm 3.5$ & 0.46 \\
BMl (kg/m²) & $21.9 \pm 2.0$ & $22.1 \pm 2.1$ & 0.47 \\
Duration of infertility (years) & $4.4 \pm 0.43$ & $3.8 \pm 0.41$ & 0.52 \\
Primary infertility $n$ (\%) & $54(30.9)$ & $40(25.2)$ & 0.25 \\
Basal FSH (IU/L) & $12.8 \pm 5.1$ & $10.2 \pm 3.2$ & $<0.01$ \\
AMH (ng/ml) & $0.3 \pm 0.02$ & $0.7 \pm 0.02$ & $<0.01$ \\
AFC ( $n)$ & $2.3 \pm 0.12$ & $3.1 \pm 0.09$ & 0.22 \\
FORT (\%) & $65 \pm 4.6$ & $75 \pm 3.7$ & 0.01 \\
FOl (\%) & $60 \pm 5.7$ & $78 \pm 5.2$ & 0.01 \\
Day 2 embryos $(n)$ & $0.8 \pm 0.08$ & $1.4 \pm 0.12$ & $<0.01$ \\
Top-quality embryos $(n)$ & $0.6 \pm 0.08$ & $0.9 \pm 0.12$ & 0.06 \\
\hline
\end{tabular}

in 175 patients with mild stimulation and 159 patients with conventional GnRH antagonist.

\section{Overall Comparison of the Two Protocols (Table 1)}

Baseline characteristics of patients such as age, BMI, infertility duration, rate of primary infertility, and AFC (antral follicle count) were not significantly different between the two protocols. However, basal FSH of patients treated with mild stimulation was significantly higher compared with that of patients treated with conventional stimulation $(p<0.01)$; Similarly, $\mathrm{AMH}$ concentration of patients that used mild ovarian stimulation was significantly lower than that of patients who used conventional stimulation.

The overall comparison of the two regimens showed that mild ovarian stimulation was associated with a significantly lower FORT (65 \pm 46 vs. $75 \pm 37, p=0.01)$, FOI (60 \pm 57 vs. $78 \pm 52, p=0.01$ ), number of day-2 embryo $(0.8 \pm 0.8$ vs. $1.4 \pm 1.2, p \leq 0.01)$, as compared with conventional stimulation.

\section{Analysis According to the AFC}

As the outcome between the two regimens was different, a subsequent analysis was performed according to the AFC in order to identify a subgroup of patients with no difference in cycle outcome. It did appear that no difference actually existed in the subgroup of patients with $\mathrm{AFC}<3$.

Table 2 shows the baseline characteristics and the cycle outcome of patients separated according to the AFC and the protocol used. In AFC < 3 group, 106 out of 140 (75.7\%) patients received a mild stimulation and 34 (24.3\%) a conventional one. In $\mathrm{AFC} \geq 3$ group, 69 out of 194 (35.5\%) patients and $125(64.5 \%)$ patients received, respectively, mild and conventional stimulations.

Regarding primary end points, no significant difference in FORT $(p=0.71)$, FOI $(p=0.12)$, day 2 embryos $(p=0.052)$ and in the number of good embryos $(p=0.26)$ could be observed between mild and conventional stimulation in $\mathrm{AFC}<3$ group. In contrast, in $\mathrm{AFC} \geq 3$ group, mild ovarian stimulation was associated with a significantly lower FORT (42 \pm 3.0 vs. $73 \pm 4.3, p<0.01)$, FOI $(38 \pm 3.2$ vs. $73 \pm 4.3$, $p<0.01)$, number of day-2 embryo 2 (0.9 \pm 0.01 vs. 1.5 $\pm 0.01 ; p<0.01)$, number of good embryo $(0.9 \pm 0.01$ vs. $1.5 \pm 0.01 ; p<0.01)$ as compared with conventional stimulation (Table 2).

All covariates were screened through the univariate regression model between continuous outcomes (FORT, FOI, Day 2 embryos and Top-quality embryos) with mild vs. conventional stimulation protocols. The final model of multiple linear regression included the biologically significant factors after removing the weakest factors by using backward stepwise regression approach.

The results in Table 3 showed that, in group AFC $<3$, stimulation protocol (mild vs. conventional) had a significant impact on the FOI $(p<0.05)$, but not on the FORT, Day 2 embryos and Top-quality embryos $(p>0.05)$. No confounding factor was found in the multiple linear regression models. In group $\mathrm{AFC} \geq 3$, stimulation protocol (mild vs. conventional) had significant influence on all primary end points, including FOI, FORT, Day 2 embryos and Topquality embryos $(p<0.05)$. In the modeling, only BMI was detected as a potential confounding factor to influence the relationship between stimulation protocol and Top-quality embryos $(p<0.05)$.

As shown in Table 4, the duration of stimulation was not significantly different between the two protocols whatever the $\mathrm{AFC}<3(p=0.39)$ or AFC $\geq 3(p=0.6)$. However, the total FSH dose required for mild ovarian stimulation was significantly lower than that required for the conventional stimulation (352 \pm 13.9 vs. $3174 \pm 66.5, p<0.01$, in $\mathrm{AFC}<3$ group; 474 \pm 33.4 vs. $3261 \pm 59.1, p<0.01$, in $\mathrm{AFC} \geq 3$ group. The fertilization rate observed with the two regimens was similar, independently of the AFC count. Regarding the cancellation rate, it was unable to measure a statistical difference between the two regimens in patients with $\mathrm{AFC} \geq 3$ due to sample size $<5$. Moreover, in $\mathrm{AFC}<3$ group, the cancellation rate was insignificantly different in the conventional stimulation vs. in the mild ovarian stimulation $[10(22.7 \%)$ vs. $10(8.7 \%)$, $p=0.39$ ] (Table 4).

Table 5 displays the follow up for pregnancy after embryo transfer till the time of data collecting. Among 102 patients, 30 patients underwent the mild ovarian stimulation and 72 patients the conventional stimulation. There was no significant difference in pregnancy outcomes between two protocols regardless of AFC. The percentage of patients with positive $\beta$-hCG as well as the rate of biochemical pregnancy, clinical pregnancy, early pregnancy loss and ongoing pregnancy were similar between the two stimulation regimens.

\section{DISCUSSION}

This retrospective study allows to conclude that, in Poseidon group 4 patients, both ovarian stimulation regimens results in similar outcomes when the AFC is $<3$. In contrast, the GnRH 
TABLE 2 | Baseline characteristics and outcome of patients according the AFC analysis.

\begin{tabular}{|c|c|c|c|c|c|c|}
\hline & \multicolumn{3}{|c|}{ AFC $<3$} & \multicolumn{3}{|c|}{$A F C \geq 3$} \\
\hline Age (yrs) & $42.8 \pm 3.6$ & $41.7 \pm 3.6$ & 0.18 & $41 \pm 3.5$ & $40.7 \pm 3.4$ & 0.67 \\
\hline $\mathrm{BMI}\left(\mathrm{kg} / \mathrm{m}^{2}\right)$ & $21.8 \pm 1.9$ & $22.4 \pm 2.4$ & 0.28 & $22 \pm 2.1$ & $22 \pm 2.0$ & 0.93 \\
\hline Duration of infertility (years) & $4.7 \pm 0.46$ & $4.2 \pm 0.8$ & 0.58 & $3.8 \pm 0.4$ & $3.6 \pm 0.3$ & 0.83 \\
\hline AMH (ng/ml) & $0.3 \pm 0.02$ & $0.6 \pm 0.04$ & $<0.01$ & $0.4 \pm 0.02$ & $0.7 \pm 0.02$ & $<0.01$ \\
\hline $\mathrm{AFC}(n)$ & $1.5 \pm 0.7$ & $1.7 \pm 0.6$ & 0.24 & $3.5 \pm 0.5$ & $3.5 \pm 0.5$ & 0.21 \\
\hline FORT (\%) & $79 \pm 5.1$ & $82 \pm 7.2$ & 0.71 & $42 \pm 3.0$ & $73 \pm 3.2$ & $<0.01$ \\
\hline FOI (\%) & $74 \pm 6.7$ & $95 \pm 11.2$ & 0.12 & $38 \pm 3.2$ & $73 \pm 4.3$ & $<0.01$ \\
\hline Day 2 embryos (n) & $0.8 \pm 0.08$ & $1.1 \pm 0.17$ & 0.052 & $0.9 \pm 0.01$ & $1.5 \pm 0.01$ & $<0.01$ \\
\hline
\end{tabular}

TABLE 3 | Multiple linear regression analysis of FORT, FOI, Day 2 embryos and Top-quality embryos with mild versus conventional stimulation protocols.

\begin{tabular}{|c|c|c|c|c|c|c|c|c|}
\hline Covariate & \multicolumn{4}{|c|}{$\mathrm{AFC}<3$} & \multicolumn{4}{|c|}{$A F C \geq 3$} \\
\hline FORT as a continuous outcome & & & & & & & & \\
\hline Stimulation protocol (mild vs. conventional) & 0.01 & 0.11 & 0.06 & 0.949 & 0.26 & 0.06 & 4.47 & $<0.01$ \\
\hline Age in year & -0.02 & 0.01 & -1.44 & 0.15 & $\mathrm{~N} / \mathrm{A}$ & $N / A$ & N/A & $\mathrm{N} / \mathrm{A}$ \\
\hline Duration of infertility & $\mathrm{N} / \mathrm{A}$ & $N / A$ & N/A & $\mathrm{N} / \mathrm{A}$ & -0.01 & 0.01 & -1.60 & 0.11 \\
\hline FSH day 2 & 0.026 & 0.01 & 2.64 & 0.09 & $\mathrm{~N} / \mathrm{A}$ & $N / A$ & N/A & $\mathrm{N} / \mathrm{A}$ \\
\hline \multicolumn{9}{|l|}{ FOI as a continuous outcome } \\
\hline Stimulation protocol (mild vs conventional) & 0.304 & 0.13 & 2.28 & 0.024 & 0.403 & 0.07 & 6.08 & $<0.01$ \\
\hline FSH day 2 & 0.04 & 0.01 & 3.15 & 0.002 & 0.013 & 0.01 & 1.93 & 0.06 \\
\hline \multicolumn{9}{|c|}{ Number of day 2 embryos as a continuous outcome } \\
\hline Stimulation protocol (mild vs. conventional) & 0.17 & 0.15 & 1.14 & 0.26 & 0.35 & 0.14 & 2.55 & 0.012 \\
\hline BMl & $\mathrm{N} / \mathrm{A}$ & $N / A$ & $\mathrm{~N} / \mathrm{A}$ & $\mathrm{N} / \mathrm{A}$ & 0.097 & 0.03 & 3.06 & 0.003 \\
\hline
\end{tabular}

N/A, not applicable.

TABLE 4 | Secondary end points of two ovarian stimulation regimens.

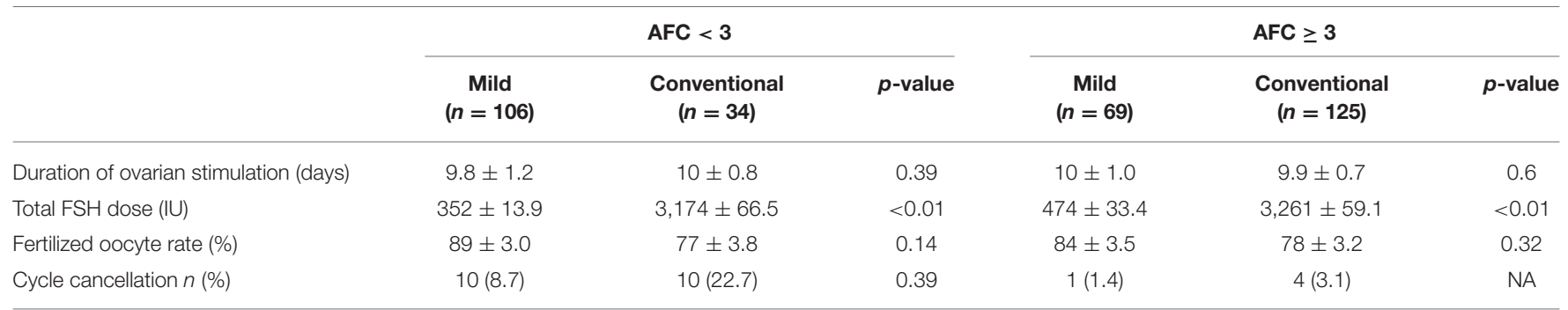

N/A, not applicable. 
TABLE 5 | Cycle outcomes following the two stimulation protocols.

\begin{tabular}{lccc}
\hline & $\begin{array}{c}\text { Mild } \\
(\boldsymbol{n}=\mathbf{3 0})\end{array}$ & $\begin{array}{c}\text { Antagonist } \\
(\boldsymbol{n}=\mathbf{7 2})\end{array}$ & $\begin{array}{c}\mathbf{R R} \\
(\mathbf{9 5 \%} \mathbf{C l})\end{array}$ \\
\hline$\beta$-hCG & $7(23.3 \%)$ & $24(33.3 \%)$ & $0.7(0.34-1.45)$ \\
Biochemical pregnancy & $2(6.7 \%)$ & $8(11 \%)$ & $0.6(0.14-2.66)$ \\
Clinical pregnancy & $5(16.7 \%)$ & $16(22.2 \%)$ & $0.75(0.30-1.86)$ \\
Early pregnancy loss & $1(3.3 \%)$ & $6(8.3 \%)$ & $0.4(0.05-3.18)$ \\
Ongoing pregnancy & $4(13.3 \%)$ & $10(13.9 \%)$ & $0.96(0.33-2.82)$ \\
\hline
\end{tabular}

conventional antagonist protocol with maximum initial FSH dose (300-375 IU/day) and supplementary LH (75-150 IU/day) is more effective than the mild one when $\mathrm{AFC}$ is $\geq 3$. Our results are in good accordance with the concept that not all poor responders are the same (25).

While it has been well demonstrated that a poor ovarian response is associated with the decrease in the IVF success rate $(4,5)$, it is still unclear to define the optimal strategy or protocol in that situation $(12,13)$. Studies comparing mild stimulation protocols with conventional GnRH agonist or antagonist regimens could not demonstrate a substantial difference in terms of live birth rate but a significant improvement in the cost effectiveness (22-24).

One of the major issues clearly remains the consensus regarding the definition of the POR. Unfortunately, Bologna criteria could not fully eliminate the heterogeneity in the patients'profile and did not take into account several medical and genetic risk factors (26). Consequently, conflicting results were reported when mild and conventional stimulations were compared: some studies found no difference between the two regimens $(27,28)$, while, in others, the outcome of mild stimulation was lower (29-31).

Subsequently, Poseidon's stratification aimed at identifying patients according to the prognosis, women of Group 4 being characterized by a very low prognosis. In this context, to the best of our knowledge, no comparative studies have been performed until now between conventional and mild stimulations in Group 4 poor responders. Therefore, the purpose of our study was set up a comparative analysis on the effects of mild and convention stimulation in this subgroup of women. In order to assess the ovarian sensitivity to FSH, the end points were the index FORT and FOI which both reflect the dynamic response to FSH and the efficacy of the stimulation regimen $(18,19)$.

Due to the retrospective nature of the study, some baseline characteristics significantly differed between the two treated groups. Significant differences in the concentrations of basal FSH and AMH were observed attesting that patients in mild stimulation group tend to have a lower ovarian reserve as compared to those treated with conventional stimulation.

Interestingly, the relative efficacy of each stimulation depends on the ovarian reserve. After controlling the impact of confounding factors, our results show that, in women characterized by a low AFC $(<3)$, the efficacy of the two regimens assessed by FORT, number of embryo day 2, and number of good quality embryos were not significantly different, except for FOI. In contrast, in women characterized by a high AFC ( $\geq 3)$, the efficacy of the two regimens assessed by FORT, FOI, number of embryo day 2, and number of good quality embryos significantly differed from each other. In addition, BMI was found to be the only potential confounding factor between the two regimens and the number of Top-quality embryos. This observation could be explained by the relationship between high BMI and alterations of serum metabolic parameters (increase in serum fatty acid levels) and of follicular fluid composition. As oocytes and embryos are quite sensitive to changes in their microenvironment, adverse effects of obesity on conception has been suggested (32). Furthermore, high free fatty acids levels and changes in adipokines associated with high BMI seems to affect oocyte competence (33). Finally, high BMI induces insulin resistance and hyperinsulinemia which stimulates both steroidogenesis and luteinizing hormone ( $\mathrm{LH})$ receptor expression in ovarian theca and granulosa cells. As a result, ovulation and oocyte maturation in obese women may be affected by the overexpression of LH (34).

Consequently, as the amount of total FSH dose was dramatically higher when using the conventional protocol, these results allow us to recommend a mild stimulation, the most cost-effective regimen in these patients with a very poor ovarian reserve. In contrast, in women with baseline $\mathrm{AFC} \geq 3$, our study shows when applying conventional protocol, the primary end points the (FORT ratio, FOI, number of embryo day 2 and number of good quality embryo) were significantly better as compared to the mild stimulation. These findings indicate that increasing the dose of FSH above a certain threshold allows to stimulate follicular recruitment, maturation and to achieve better outcomes. It has been shown that the optimal daily dose of FSH is likely to be about $300 \mathrm{IU}$ and the addition of $\mathrm{LH}$ might improve the ovarian response to $\mathrm{FSH}$, specifically in patients with moderate to severe forms of ovarian insufficiency (35). Presumably, as LH acts to stimulate theca cell androgen production, the number of FSH receptors increase at the granulosa cell levels and the sensitivity to FSH is actually improved.

In patients belonging to Poseidon group 4, the strategies currently support the use of a standard ovarian stimulation with maximum starting dose of FSH and adjuvant LH. We agree with this strategy when AFC is $\geq 3$ because this regimen is more effective than the mild stimulation protocol. Nevertheless, when AFC is $<3$, the mild ovarian stimulation seems more friendly and less costly with similar outcome.

While our study was not designed to assess the pregnancy rate as a main end point, we observed that the cycle outcome was at least identical to that reported in previous studies, even though the selection criteria were very strict. The ongoing pregnancy rate was equivalent with the result of Youssef et al. (36) but remarkably higher than of Klinkert et al. (15). However, all these studies similarly concluded to the absence of difference in cycle outcome between the two protocols. In our study, biochemical pregnancy, early pregnancy loss, clinical pregnancy rates were similar in both treatment groups, in accordance with other 
comparable studies $(27-31,37)$. A recent guideline of ASRM has claimed the lack of difference in clinical pregnancy rate between the two protocols while we need further studies to conclude on other outcomes, such as cumulative pregnancy rate and live birth rate (22).

The strength of this study is the way of assessing the ovarian response to FSH by using the index FORT and FOI. This new approach allows to evaluate ovarian stimulation efficiency more accurately by reporting the number of growing follicles or oocytes to the basal AFC.

The weakness of the study is related to insufficient sample size, however the number of patients involved in this study was an overall number of patients admitted to the hospital during the study period. Besides, the study also faced to the information bias because of using retrospective data and selection bias due to no-randomized selection. The limited number of patients included in this study was insignificant to analyze the cumulative pregnancy rate and live birth rate. Furthermore, a cost effectiveness analysis should be done in further studies aimed to measure the huge difference between the total amount of FSH in mild vs. conventional stimulation, and assess the effectiveness of higher doses of gonadotrophins in the mild stimulation protocol. Because, it clearly indicated that a mild stimulation is associated with a lower consumption of FSH.Z.

\section{REFERENCES}

1. Sobotka T, Beaujouan É. Late Motherhood in Low-Fertility Countries: Reproductive Intentions, Trends and Consequences. Preventing Age Related Fertility Loss. Cham: Springer International Publishing (2018). p. 11-29.

2. te Velde ER, Pearson PL. The variability of female reproductive ageing. Hum Reprod Update. (2002) 8:141-154. doi: 10.1093/humupd/8.2.141

3. O'Flynn N. Assessment and treatment for people with fertility problems: NICE guideline. Br J Gen Pract. (2014) 64:50-1. doi: 10.3399/bjgp14X676609

4. van Loendersloot LL, van Wely M, Limpens J, Bossuyt PMM, Repping $\mathrm{S}$, van der Veen F. Predictive factors in in vitro fertilization (IVF): a systematic review and meta-analysis. Hum Reprod Update. (2010) 16:57789. doi: 10.1093/humupd/dmq015

5. Broer SL, van Disseldorp J, Broeze KA, Dolleman M, Opmeer BC, Bossuyt $\mathrm{P}$, et al. Added value of ovarian reserve testing on patient characteristics in the prediction of ovarian response and ongoing pregnancy: an individual patient data approach. Hum Reprod Update. (2013) 19:2636. doi: 10.1093/humupd/dms041

6. Serour G, Mansour R, Serour A, Aboulghar M, Amin Y, Kamal O, et al. Analysis of 2,386 consecutive cycles of in vitro fertilization or intracytoplasmic sperm injection using autologous oocytes in women aged 40 years and above. Fertil Steril. (2010) 94:1707-12. doi: 10.1016/j.fertnstert.2009.09.044

7. Gunby J, Bissonnette F, Librach C, Cowan L, IVF Directors Group of the Canadian Fertility and Andrology Society. Assisted reproductive technologies (ART) in Canada: 2007 results from the Canadian ART Register. Fertil Steril. (2011) 95:542-7.e1-10. doi: 10.1016/j.fertnstert.2010.05.057

8. Sunkara SK, Rittenberg V, Raine-Fenning N, Bhattacharya S, Zamora J, Coomarasamy A. Association between the number of eggs and live birth in IVF treatment: an analysis of 400135 treatment cycles. Hum Reprod. (2011) 26:1768-74. doi: 10.1093/humrep/der106

9. Polyzos NP, Devroey P. A systematic review of randomized trials for the treatment of poor ovarian responders: is there any light at the end of the tunnel? Fertil Steril. (2011) 96:105861.e7. doi: 10.1016/j.fertnstert.2011.09.048

10. Ferraretti AP, La Marca A, Fauser BCJM, Tarlatzis B, Nargund G, Gianaroli L, et al. ESHRE consensus on the definition of 'poor response' to ovarian

\section{CONCLUSION}

In women classified in Poseidon group 4, the comparative analysis of mild and conventional GnRH antagonist stimulation should be analyzed according to the baseline AFC. If the ovarian reserve is very low (AFC $<3$ ), the efficacy between the two protocols is equivalent. Therefore, mild stimulation should be privileged, being more friendly and less costly than the GnRH antagonist protocol. In contrast, if the AFC is $\geq 3$, the conventional GnRH antagonist protocol should be firstly considered with maximum starting dose of FSH (300$375 \mathrm{IU} /$ day) and adjuvant LH (75-150 IU/day) as it seems more effective.

\section{DATA AVAILABILITY STATEMENT}

The raw data supporting the conclusions of this article will be made available by the authors, without undue reservation.

\section{AUTHOR CONTRIBUTIONS}

HL, DN, AC, HN, DT, and TL conserved and realized this study. $\mathrm{J}-\mathrm{NH}$ reviewed the manuscript. All authors contributed to the article and approved the submitted version.

stimulation for in vitro fertilization: the Bologna criteria. Hum Reprod. (2011) 26:1616-24. doi: 10.1093/humrep/der092

11. Poseidon Group (Patient-Oriented Strategies Encompassing IndividualizeD Oocyte Number), Alviggi C, Andersen CY, Buehler K, Conforti A, De Placido $\mathrm{G}$, et al. A new more detailed stratification of low responders to ovarian stimulation: from a poor ovarian response to a low prognosis concept. Fertil Steril. (2016) 105:1452-3. doi: 10.1016/j.fertnstert.2016.02.005

12. Ubaldi F, Vaiarelli A, D’Anna R, Rienzi L. Management of poor responders in IVF: is there anything new? Biomed Res Int. (2014) 2014:352098. doi: 10.1155/2014/352098

13. Patrizio P, Vaiarelli A, Levi Setti PE, Tobler KJ, Shoham G, Leong M, et al. How to define, diagnose and treat poor responders? Responses from a worldwide survey of IVF clinics. Reprod Biomed Online. (2015) 30:581592. doi: 10.1016/j.rbmo.2015.03.002

14. Berkkanoglu $\mathrm{M}$, Ozgur $\mathrm{K}$. What is the optimum maximal gonadotropin dosage used in microdose flare-up cycles in poor responders? Fertil Steril. (2010) 94:662-5. doi: 10.1016/j.fertnstert.2009.03.027

15. Klinkert ER, Broekmans FJM, Looman CWN, Habbema JDF, te Velde ER. Expected poor responders on the basis of an antral follicle count do not benefit from a higher starting dose of gonadotrophins in IVF treatment: a randomized controlled trial. Hum Reprod. (2005) 20:611-5. doi: 10.1093/humrep/deh663

16. Land JA, Yarmolinskaya MI, Dumoulin JC, Evers JL. High-dose human menopausal gonadotropin stimulation in poor responders does not improve in vitro fertilization outcome. Fertil Steril. (1996) 65:9615. doi: 10.1016/S0015-0282(16)58269-7

17. Karande VC, Jones GS, Veeck LL, Muasher SJ. High-dose follicle-stimulating hormone stimulation at the onset of the menstrual cycle does not improve the in vitro fertilization outcome in low-responder patients. Fertil Steril. (1990) 53:486-9. doi: 10.1016/S0015-0282(16)53345-7

18. Grynberg M, Labrosse J. Understanding follicular output rate (FORT) and its implications for POSEIDON criteria. Front Endocrinol. (2019) 10:246. doi: 10.3389/fendo.2019.00246

19. Alviggi C, Conforti A, Esteves SC, Vallone R, Venturella R, Staiano S, et al. Understanding ovarian hypo-response to exogenous gonadotropin in ovarian stimulation and its new proposed marker-the follicle-to-oocyte (FOI) index. Front Endocrinol. (2018) 9:589. doi: 10.3389/fendo.2018.00589 
20. Vaiarelli A, Cimadomo D, Ubaldi N, Rienzi L, Ubaldi FM. What is new in the management of poor ovarian response in IVF?. Curr Opin Obstet Gynecol. (2018) 30:155-162. doi: 10.1097/GCO.0000000000000452

21. Humaidan P, La Marca A, Alviggi C, Esteves SC, Haahr T. Future perspectives of POSEIDON stratification for clinical practice and research. Front Endocrinol. (2019) 10:439. doi: 10.3389/fendo.2019.00439

22. Practice Committee of the American Society for Reproductive Medicine. Electronic address: ASRM@asrm.org. Comparison of pregnancy rates for poor responders using IVF with mild ovarian stimulation versus conventional IVF: a guideline. Fertil Steril. (2018) 109:993-99. doi: 10.1016/j.fertnstert.2018.03.019

23. Griesinger G, Diedrich K, Tarlatzis BC, Kolibianakis EM. GnRHantagonists in ovarian stimulation for IVF in patients with poor response to gonadotrophins, polycystic ovary syndrome, and risk of ovarian hyperstimulation: a meta-analysis. Reprod Biomed Online. (2006) 13:628-38. doi: 10.1016/S1472-6483(10)60652-9

24. $\mathrm{Pu} \mathrm{D}, \mathrm{Wu}$ J, Liu J. Comparisons of $\mathrm{GnRH}$ antagonist versus $\mathrm{GnRH}$ agonist protocol in poor ovarian responders undergoing IVF. Hum Reprod. (2011) 26:2742-9. doi: 10.1093/humrep/der240

25. Polyzos NP, Popovic-Todorovic B. SAY NO to mild ovarian stimulation for all poor responders: it is time to realize that not all poor responders are the same. Hum Reprod. (2020) 35:1964-71. doi: 10.1093/humrep/deaal83

26. Younis JS, Ben-Ami M, Ben-Shlomo I. The Bologna criteria for poor ovarian response : a contemporary critical appraisal. J Ovarian Res. (2015) 8:76. doi: 10.1186/s13048-015-0204-9

27. Goswami SK, Das T, Chattopadhyay R, Sawhney V, Kumar J, Chakravarty BN, et al. A randomized single-blind controlled trial of letrozole as a low-cost IVF protocol in women with poor ovarian response: a preliminary report. Hum Reprod. (2004) 19:2031-5. doi: 10.1093/humrep/deh359

28. Bastu E, Buyru F, Ozsurmeli M, Demiral I, Dogan M, Yeh J. A randomized, single-blind, prospective trial comparing three different gonadotropin doses with or without addition of letrozole during ovulation stimulation in patients with poor ovarian response. Eur J Obstet Gynecol Reprod Biol. (2016) 203:304. doi: 10.1016/j.ejogrb.2016.05.027

29. Revelli A, Chiadò A, Dalmasso P, Stabile V, Evangelista F, Basso G, et al. "Mild" vs. "long" protocol for controlled ovarian hyperstimulation in patients with expected poor ovarian responsiveness undergoing in vitro fertilization (IVF): a large prospective randomized trial. J Assist Reprod Genet. (2014) 31:809-815. doi: 10.1007/s10815-014-0227-y

30. Mohsen IA, El Din RE. Minimal stimulation protocol using letrozole versus microdose flare up GnRH agonist protocol in women with poor ovarian response undergoing ICSI. Gynecol Endocrinol. (2013) 29:1058. doi: 10.3109/09513590.2012.730569

31. Pilehvari S, ShahrokhTehraninejad E, Hosseinrashidi B, Keihkhah F, Haghollahi F, Aziminekoo E. Comparison pregnancy outcomes between minimal stimulation protocol and conventional $\mathrm{GnRH}$ antagonist protocols in poor ovarian responders. J Family Reprod Health. (2016) 10:35-42.

32. Valckx SDM, De Pauw I, De Neubourg D, Inion I, Berth M, Fransen E, et al. BMI-related metabolic composition of the follicular fluid of women undergoing assisted reproductive treatment and the consequences for oocyte and embryo quality. Hum Reprod. (2012) 27:3531-9. doi: 10.1093/humrep/des350

33. Brewer CJ, Balen AH. The adverse effects of obesity on conception and implantation. Reproduction. (2010) 140:347-364. doi: 10.1530/REP-09-0568

34. Jain A, Polotsky AJ, Rochester D, Berga SL, Loucks T, Zeitlan G et al. Pulsatile luteinizing hormone amplitude and progesterone metabolite excretion are reduced in obese women. J Clin Endocrinol Metab. (2007) 92:246873. doi: $10.1210 /$ jc.2006-2274

35. Humaidan P, Chin W, Rogoff D, D’Hooghe T, Longobardi S, Hubbard J, et al. Efficacy and safety of follitropin alfa/lutropin alfa in ART: a randomized controlled trial in poor ovarian responders. Hum Reprod. (2017) 32:54455. doi: 10.1093/humrep/dew360

36. Youssef MA, van Wely M, Al-Inany H, Madani T, Khodabakhshi S, Alhalabi $\mathrm{M}$, et al. A mild ovarian stimulation strategy in women with poor ovarian reserve undergoing IVF: a multicenter randomized non-inferiority trial. Hum Reprod. (2017) 32:112-8. doi: 10.1093/humrep/dew282

37. Ubaldi F, Rienzi L, Baroni E, Ferrero S, Iacobelli M, Minasi MG, et al. Hopes and facts about mild ovarian stimulation. Reprod Biomed Online. (2007) 14:675-81. doi: 10.1016/S1472-6483(10)60667-0

Conflict of Interest: The authors declare that the research was conducted in the absence of any commercial or financial relationships that could be construed as a potential conflict of interest.

Copyright (c) 2020 Le, Nguyen, Cao, Nguyen, Tham, Le and Hugues. This is an open-access article distributed under the terms of the Creative Commons Attribution License (CC BY). The use, distribution or reproduction in other forums is permitted, provided the original author(s) and the copyright owner(s) are credited and that the original publication in this journal is cited, in accordance with accepted academic practice. No use, distribution or reproduction is permitted which does not comply with these terms. 